\title{
Using Carbon Laser Patterning to Produce Flexible, Metal-Free Humidity Sensors
}

\author{
Simon Delacroix, Anna Zieleniewska, Andrew J. Ferguson, Jeffrey L. Blackburn, Sebastian Ronneberger, \\ Felix F. Loeffler, and Volker Strauss*
}

Cite This: ACS Appl. Electron. Mater. 2020, 2, 4146-4154

Read Online

ABSTRACT: A relative humidity sensor was produced by carbon laser patterning of a carbon precursor ink on a flexible substrate. Citric acid and urea, both inexpensive and naturally abundant molecules, are used as initial precursors to obtain a porous carbon foam after $\mathrm{CO}_{2}$ laser irradiation. The laser-patterned material is characterized by electron microscopy, Raman spectroscopy, and vertical scanning interferometry. An intrinsic p-type semiconducting behavior was confirmed by thermoelectric and Hall measurements. The resistance of this porous, metal-free material is sensitive to atmospheric variations, namely, temperature and relative humidity $(\approx 5 \Omega \cdot \%)$. Under dry atmosphere, the sensor

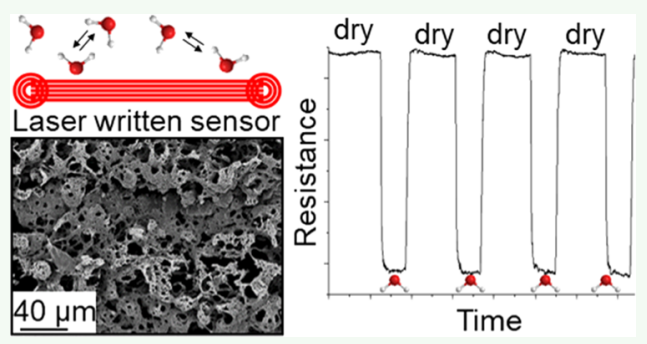
acts as a thermometer with a linear relationship between temperature and relative variation of resistance $\left(0.07 \% \cdot \mathrm{K}^{-1}\right)$. The evolution of the sensor resistance at different relative humidities and temperatures is studied by electrical impedance measurements. The kinetic transitory regime of water desorption from the carbonaceous surface of the sensor is analyzed using Langmuir's model. The equilibrium constant of adsorption $K_{\text {ads }}$ has been determined, and the standard enthalpy of adsorption of water on the sensor surface is estimated at $\Delta_{\text {ads }} H^{\circ}=-42.6 \mathrm{~kJ} \cdot \mathrm{mol}^{-1}$. The simple and inexpensive production and its high, stable sensitivity make laser-patterned carbon interesting for humidity sensing applications, and the method allows for the large-scale production of printed sensor arrays.

KEYWORDS: humidity sensor, carbon laser patterning, laser carbonization, carbon semiconductor, laser-induced graphene, sensing

\section{INTRODUCTION}

The development of new lab-on-a-chip sensors is essential to move toward miniaturization of devices, realization of sensor arrays, and their applications for on-site analysis. ${ }^{1}$ Various fields could benefit from such progress, especially public health, with the sensing of volatile organic compounds or biomolecules for disease diagnosis. ${ }^{2-4}$ Such future sensors have to be inexpensive, easily producible, simple to integrate into a device, and based on ecologically sustainable precursors. The flexibility is another desired characteristic, which can assure a larger versatility of use of these lab-on-a-chip sensors. ${ }^{5}$

In terms of humidity sensing, the most common commercial materials used today are based on metal oxides or porous silicon. ${ }^{6}$ To reach the above-mentioned specifications of a sensor, however, metal-free materials and, in particular, conductive carbon materials are prime candidates. ${ }^{7}$ In recent years, carbon materials, such as graphene and carbon nanotubes, were subject to detailed investigations as active materials for humidity sensing. ${ }^{8,9}$ Their high electronic mobility at room temperature and small dimensions make graphenes, carbon nanotubes, and their derivatives extremely sensitive to molecules present in their environment. ${ }^{10,11}$ Advantages in terms of simple production, miniaturization, eco-friendliness, and economical fairness were targeted. The carbon nanoallotropes served as excellent test beds for mechanistic studies; however, their real-world application is still impeded by the tedious production and resulting high costs. Here, use of bio-based carbon provides a significant advantage in terms of production costs. ${ }^{12}$ Moreover, production techniques for realizing sensor array arrangements are in demand, as these help to reduce measurement errors, cross-interference, and the issue of normalization. ${ }^{13,14}$

In the past years, printed carbon foams have been shown to be very promising as sensors due to their high specific surface area. ${ }^{15,16} \mathrm{~A}$ hurdle to the development of new devices is the difficulty to process and integrate such carbon foams into electronic circuits. High-temperature treatments in ovens and purification steps with hazardous solution are often used during their syntheses. ${ }^{17}$ Within the last decade, a simple processing technique for carbon foams has been developed: direct laser writing of carbons, also referred to as laser-induced carbons. ${ }^{18-20}$ In general, this strategy of laser fabrication has many advantages such as on-spot production, low energy

Received: October 23, 2020

Accepted: November 23, 2020

Published: December 9, 2020

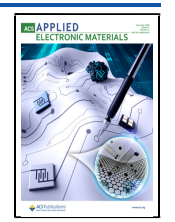




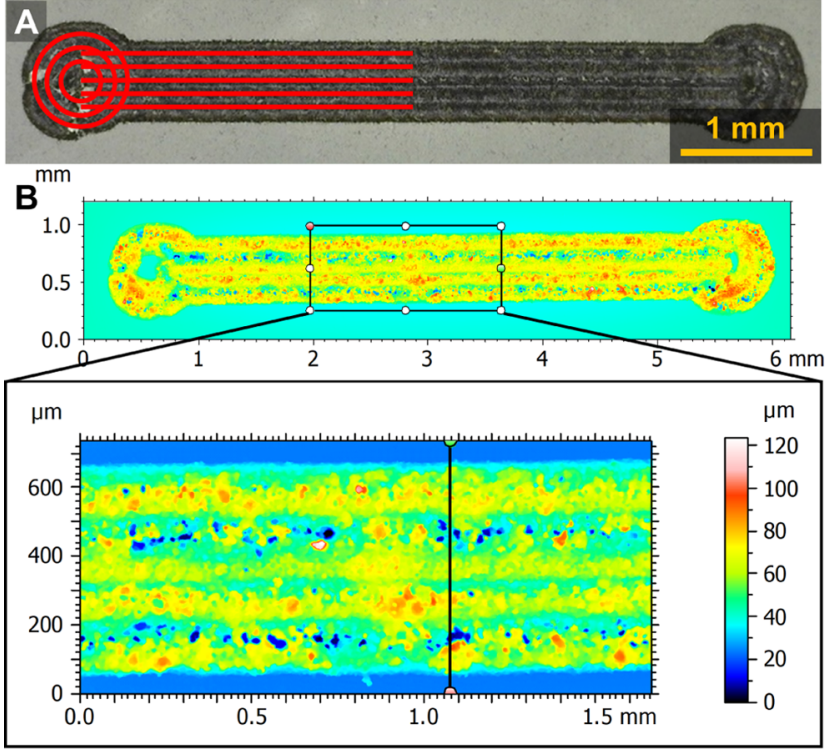

C

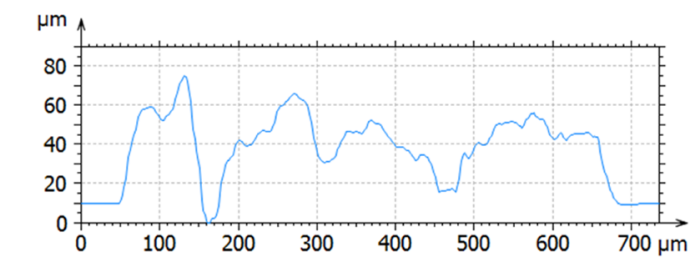

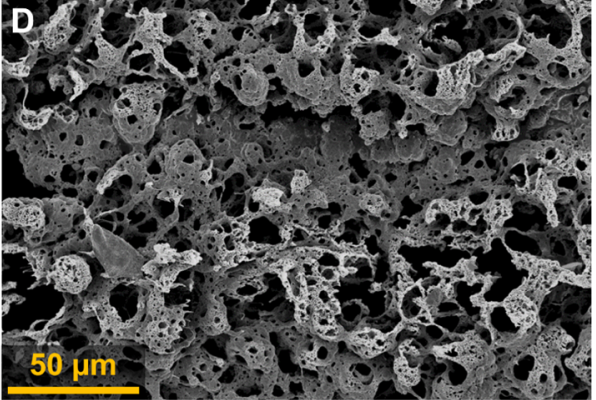

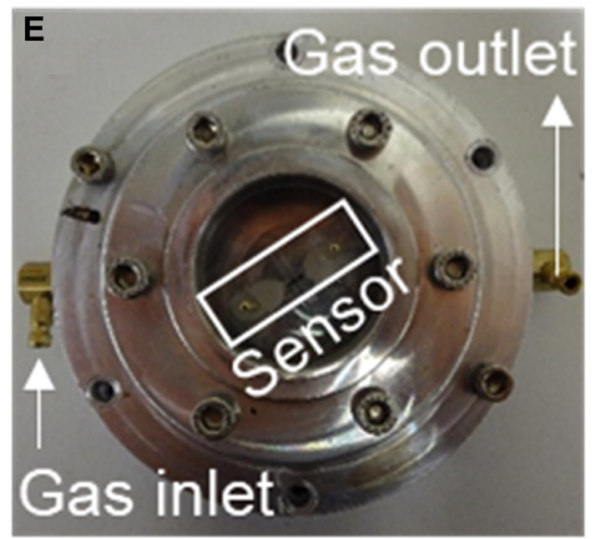

Figure 1. (A) Digital micrograph of the sensor platform obtained by laser patterning with the programmed laser pattern overlaid (red); (B) vertical scanning interferometry of the selected part of the digital micrograph; (C) height profile of the sensor platform on a line perpendicular to the laser pattern; (D) scanning electron micrograph of the sensor; and (E) sensor in the atmospheric chamber with gas inlet and outlet to control the relative humidity.

consumption, and high precision. Furthermore, laser patterning is compatible with roll-to-roll techniques, which allow a scalable production of such devices, and allows for the production of array patterns. $^{20,21}$

Typical precursors of laser-patterned carbon foams are graphene oxide (GO) or polyimide $(\mathrm{PI})^{22}$ and also other precursors like phenolic resin, ${ }^{23}$ paperboard, ${ }^{24}$ wood, ${ }^{25}$ coconut, ${ }^{26}$ and lignin ${ }^{27}$ have been studied. Recently, an alternative route to obtain laser-patterned carbon from molecule-based inks was introduced. ${ }^{28-31}$ Carbon-rich nanoparticles were synthesized via thermal treatment of organic molecules. ${ }^{32}$ These particles act as a carbon network forming agent (CNFA) in an ink, which is used to make films on various substrates. Laser patterning of the carbonaceous precursor film leads to a porous foam of carbon, while the unexposed film is simply rinsed off the substrate. A major advantage of this technique is the possibility to directly print carbon foams on any chosen substrate from rigid conductive silicon to flexible insulator plastic, which is fundamental for the production of future sensor arrays. ${ }^{32}$ Moreover, nitrogen functionalities are easily incorporated into porous carbon networks to provide additional binding sites for polar gases.

In this work, we used laser patterning of our in-housedeveloped carbon precursor ink to design a relative humidity sensor. Laser patterning of chemiresistors made of carbon foam is realized over a small area $\left(2.5 \mathrm{~mm}^{2}\right)$ on a flexible poly(ethylene terephthalate) (PET) substrate. First, the sensor platform is characterized by electron microscopy and Raman spectroscopy. Their intrinsic electronic properties, such as carrier density and mobility, are determined using thermoelectric and Hall measurements. The variation of sensor resistance with temperature is thoroughly studied, followed by systematic testing of the change of resistance under different atmospheric conditions, namely, temperature and relative humidity. Finally, the interaction between water molecules and the sensor surface was characterized by calculating the thermodynamic constants of adsorption equilibrium and the standard enthalpy of adsorption of gaseous $\mathrm{H}_{2} \mathrm{O}$ on the surface of the sensor. These results can be used to calibrate the sensor platform for quick quantitative on-spot measurements.

\section{RESULTS AND DISCUSSION}

Materials Characterization. The use of carbon-rich particles as precursors for laser-induced carbonization to obtain porous carbon foams was discussed in a previous study. ${ }^{32}$ Briefly, citric acid and urea are precarbonized in an oven at $300{ }^{\circ} \mathrm{C}$ for $2 \mathrm{~h}$ under an inert atmosphere. A black powder (CAU300) composed of carbon-rich particles and molecular side products was obtained. To eliminate the side products, the solid mixture was thoroughly washed in hot $\mathrm{H}_{2} \mathrm{O}$ (see the Experimental Section). The dry product from the washing process, referred to as CAU300p, was used as the carbon network forming agent (CNFA).

The transmission electron microscopy image of CAU300p in Figure S1B shows aggregated carbonaceous particles of diameters between 50 and $200 \mathrm{~nm}$. A peak at $2 \Theta=27.4^{\circ}$ $(\mathrm{Cu} \mathrm{K} \alpha)$ in the $\mathrm{X}$-ray diffraction pattern is characteristic of the presence of graphitic layers (Figure S1A).

The ink used for laser patterning is composed of 48 wt \% CAU300p (CNFA), 44 wt \% ethylene glycol (solvent), and 8 wt $\%$ of polyvinylpyrrolidone (film forming agent (FFA)). 
Films of thickness between 20 and $30 \mu \mathrm{m}$ (manual digital micrometer measurement) were prepared by doctor blading on PET foil, and ethylene glycol was evaporated after $10 \mathrm{~min}$ at 80 ${ }^{\circ} \mathrm{C}$.

The sensor platform was laser-patterned into the film to obtain laser-patterned carbon (LPC). To maximize the contact area with the atmosphere, the pattern is composed of five parallel lines of $5 \mathrm{~mm}$ length distributed on $0.5 \mathrm{~mm}$ width. The sensor has a geometric area of $2.5 \mathrm{~mm}^{2}$ (Figure 1A). At both ends of these line assemblies, three concentric circles were printed to ensure good electrical contact to the electrodes for impedance measurements. The nonprinted ink around the laser pattern was rinsed off with distilled water, and the sensor platform was dried under airflow.

After laser irradiation, the Raman spectrum of the LPC (Figure S2) shows a characteristic pattern of a turbostratic graphitic material: D and G vibration bands localized at 1337 and $1565 \mathrm{~cm}^{-1}$ and the $\mathrm{G}^{\prime}$ band localized at $2665 \mathrm{~cm}^{-1}$ with a full width at half-maximum of $84 \mathrm{~cm}^{-1}$. 33 This pattern, in particular, the pronounced $\mathrm{D}$ and $\mathrm{D}^{\prime}$ bands, indicates the presence of large amounts of defects in the graphitic lattice. The appearance of a semicrystalline porous structure is confirmed by transmission electron microscopy (Figure S2B). The morphology of the LPC sensor platform, probed by vertical scanning interferometry (Figure $1 \mathrm{~B}, \mathrm{C}$ ), shows high roughness with a mean height of approximately $30 \mu \mathrm{m}$ (Figure S3). A more detailed overview image of the LPC sensor platform is given in the scanning electron micrograph in Figure 1D, which shows that the LPC has a foam-like porous morphology. The presence of micropores (Figure 1D) and mesopores (Figure S2B) indicates a high surface area. The methylene blue adsorption method (Experimental Section) is well suited to determine the surface area of small quantities $(\sim 0.1 \mathrm{mg})$ of carbonaceous samples. A surface area around $238 \mathrm{~m}^{2} \cdot \mathrm{g}^{-1}$ was determined for the sensor material with reference to activated carbon $\left(1269 \mathrm{~m}^{2} \cdot \mathrm{g}^{-1}\right)$, which is a prerequisite for good sensing properties. The elemental composition was analyzed by combustion analysis of the collected laser-patterned material. The composition of $68 \% \mathrm{C}$, $13 \% \mathrm{~N}, 2 \% \mathrm{H}$, and $16 \% \mathrm{O}$ demonstrates the heterogeneity of the sensor material in the bulk. The same composition $(72 \%$ carbon, $13 \%$ nitrogen, and $14 \%$ oxygen), is also reflected in the results of the powder XPS analysis shown in Figure S4. The fits of the XPS data reveal that the majority of the carbon in the LPC is $\mathrm{sp}^{2}$-hybridized (57\%) and $\sim 18 \%$ of the carbon is $\mathrm{sp}^{3}$ hybridized. Therefore, we describe the morphology of the LPC as rather amorphous or semicrystalline. The nitrogen is predominantly incorporated in the form of pyridinic/pyrrolic or graphitic nitrogen. ${ }^{34,35}$ Notably, the bulk composition shows a strong deviation from the surface composition of $97 \% \mathrm{C}$ and $3 \% \mathrm{O}$ measured by energy-dispersive $\mathrm{X}$-ray analysis (EDX), which is due to the top-to-bottom energy input by the laser.

To obtain insights into the fundamental electronic properties of LPC films, thermoelectric and Hall effect measurements were carried out. Both experiments yielded positive values of Seebeck and Hall coefficients (Figure S5), indicating an intrinsic p-type character of the material. Notably, the values of both factors are relatively small, that is, $+2.3 \pm 0.5 \mu \mathrm{V} \cdot \mathrm{K}^{-1}$ and $+0.099 \pm 0.043 \mathrm{~cm}^{3} \cdot \mathrm{C}^{-1}$ for Seebeck and Hall coefficients, respectively. Such small positive Seebeck and Hall coefficients are not uncommon even for highly nitrogen-doped graphite or graphene materials due to unintentional doping with environmental water and oxygen molecules at room temperature. ${ }^{36,37}$
Despite the high carrier density of $7.7 \times 10^{19} \mathrm{~cm}^{-3}$, the material shows low mobilities of $0.12 \mathrm{~cm}^{2} \cdot(\mathrm{V} \cdot \mathrm{s})^{-1}$. This is in line with the presence of a large number of defects and $\mathrm{sp}^{3}$ carbon concluded from the Raman and XPS data. Similar observations have been noted for graphene where defects introduced during the nitrogen doping process are recognized to function as scattering centers that hinder the electron/hole transport. ${ }^{38,39}$ The reproducibility of these data was verified in several samples. For example, an average conductivity of 1.74 $\pm 0.29 \mathrm{~S} \mathrm{~cm}^{-1}$ was obtained for 30 sensor platforms.

To test the principle sensing properties of the laserpatterned carbon, the resistivity of the platform was measured upon variation of temperature and humidity. To this end, the platform was placed in a chamber (Figure $1 \mathrm{E}$ ), where its resistance was monitored by measuring the electrical impedance. Two electrodes separated by $5 \mathrm{~mm}$ ensured the electrical contact through the windows of this chamber. The relative humidity inside this chamber was controlled by a humidity generator with a flow of $1.3 \mathrm{~L} \cdot \mathrm{min}^{-1}$. The volume of the chamber is $\sim 0.1 \mathrm{~L}$, ensuring that a complete change of the atmosphere is achieved in about $5 \mathrm{~s}$. This system was contained and thermalized in an environmental chamber so that the relative humidity and the temperature of the sensor can be varied independently.

Resistance in Dependence of Temperature. Before changing the relative humidity inside the chamber, the resistance of the sensor was studied as a function of temperature at a constant humidity (Figure 2). To this end,

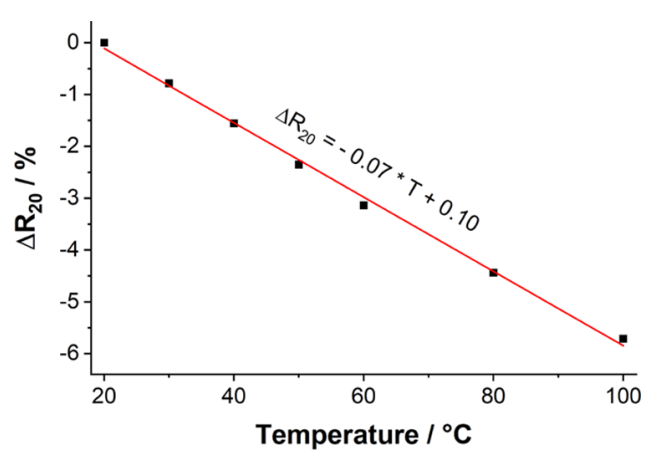

Figure 2. Relative variation of resistance in the temperature range between 20 and $100{ }^{\circ} \mathrm{C}$. The linear regression (red) is in good agreement $\left(r^{2}=0.9967\right)$ with the experimental data.

a commercial humidity generator was used, in which dry and moisture-saturated airstreams are mixed via specifically designed humidity-resistant mass flow controllers. Dry air was flushed into the chamber, while the temperature inside the chamber was increased from 20 to $100{ }^{\circ} \mathrm{C}$ in steps of $10{ }^{\circ} \mathrm{C}$. After reaching thermal equilibrium, the resistance of the sensor was measured. The frequency and the current amplitude of the electrical impedance measurements were fixed at $1000 \mathrm{~Hz}$ and $0.05 \mathrm{~mA}$, respectively, for every measurement. The relative variation of the resistance is defined by eq 1 , where $R_{20}$ and $R_{\mathrm{T}}$ are the resistance values at $20^{\circ} \mathrm{C}$ and the tested temperature $T$, respectively

$$
\Delta R_{20}=100 * \frac{R_{\mathrm{T}}-R_{20}}{R_{20}}
$$

The resistance-temperature relationship exhibits a decrease of resistance with increasing temperature. Although the commonly applied models follow an $\alpha \mathrm{e}^{1 / \mathrm{T}}$ dependence for 
thermally activated transport in a semiconductor, ${ }^{40-42}$ the weak temperature dependence suggests that it would be difficult to distinguish between these models for this material system over the tested temperature regime. However, the observed linear trend allows simple calibration of the temperature-dependent resistance of our humidity sensor. The relative resistance decrease of $0.07 \%$ per Kelvin (K) corresponds to an absolute variation of approximately $2 \Omega \cdot \mathrm{K}^{-1}$. Similar behavior is observed in other conductive organic materials such as single-walled carbon nanotubes or conducting polymers. ${ }^{42}$ The variation of resistance as a function of relative humidity in the following part is realized at constant temperatures.

Resistance in Dependence of Relative Humidity. As a first experiment to study the variation of resistance with changes in relative humidity $(\mathrm{RH})$, the sensor temperature was fixed at $40{ }^{\circ} \mathrm{C}$. The resistance was monitored during cycles between dry air and air with a relative humidity of $70 \%$ in the sensor chamber (Figure 3). The cycles of humid air were

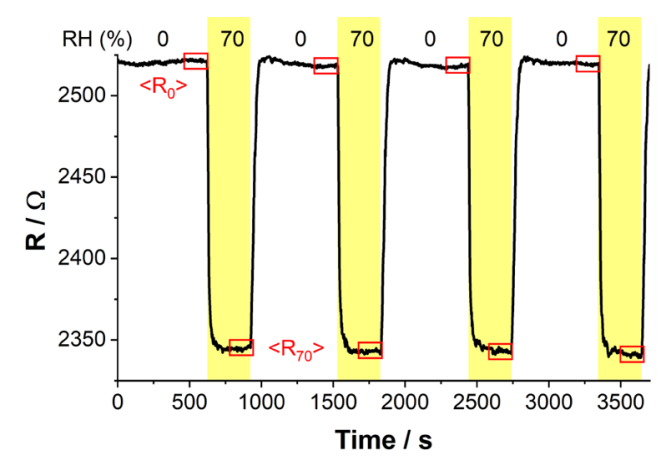

Figure 3. Variation of resistance during cycles between dry air and air with a relative humidity of $70 \%$ at $40{ }^{\circ} \mathrm{C}$. The resistance values in the red squares (50 s intervals) are used to calculate the equilibrium average resistances $\left\langle R_{0}\right\rangle$ and $\left\langle R_{70}\right\rangle$ under relative humidities of 0 and $70 \%$, respectively.

chosen to be shorter $(5 \mathrm{~min})$ than those of dry air $(10 \mathrm{~min})$, because the equilibrium is reached slower for desorption than adsorption onto the sensing platform, suggesting a moderate hydrophilicity of the material. The resistance decreases in the presence of gaseous water in the chamber, consistent with previous observations of conductivity increase in the presence of water vapor in different carbonaceous materials like carbon nanotubes, ${ }^{43}$ carbonized bamboo, ${ }^{44}$ carbon quantum dots, ${ }^{45}$ $\mathrm{N}$-doped carbon sphere, ${ }^{46}$ and graphene oxide. ${ }^{47}$ Such an increase could be explained by two phenomena: charge transfer from the water molecules to the carbon materials, increasing the number of charge carriers, ${ }^{43}$ or the occurrence of ionic conductivity in the water-filled pores of the materials for high humidity. ${ }^{48}$ Here, we postulate the presence of nitrogen and oxygen functional groups in the sensor material (13 wt \%) as polar binding sites for the adsorption of water molecules on the sensor surface even at a relatively low relatively humidity. ${ }^{49}$ Despite the lack of heteroatoms on top of the material, its porous nature allows for the penetration of gases in the environment to penetrate deep into the material and interact with functionalities. To ensure reproducible data treatment, an average of resistance values at the equilibrium was taken during the $50 \mathrm{~s}$ before a change in relative humidity for all of the cycles (red squares in Figure 3).
To study the environmental sensitivity of the sensor platform in depth, experiments were performed by varying the relative humidity and temperature (Figure 4). In a typical experiment, $10 \mathrm{~min}$ cycles between dry air and different relative humidities $(\mathrm{RH}=30-100 \%)$ at fixed temperatures $\left(10,20,30\right.$, and $\left.40{ }^{\circ} \mathrm{C}\right)$ were run. ${ }^{50}$ At 30 and $40{ }^{\circ} \mathrm{C}, 10 \mathrm{~min}$ of desorption was enough to reach equilibrium, but at 10 and 20 ${ }^{\circ} \mathrm{C}, 20 \mathrm{~min}$ of desorption is necessary. Adsorption was observed to be much faster than desorption on the sensor: the resistance reached its equilibrium value faster during the water adsorption, leading to quasi-squared shape curves.

At each temperature, the relative variation of resistance $\Delta R$ is calculated as a function of relative humidity (Figure 5) using eq 2 , where $R(\% \mathrm{RH}, T)$ is the sensor resistance in an atmosphere at temperature $T$ containing a given percentage of relative humidity

$$
\Delta R(\% \mathrm{RH}, T)=\frac{R(0, T)-R(\% \mathrm{RH}, T)}{R(0, T)}
$$

For a given temperature, the variation of resistance with relative humidity has a classic shape of an isotherm curve. These calibration curves allow us to use the sensor as a hygrometer at a given temperature. The sensitivity $S$ in $(\Omega . \%)$ of the sensor is defined as the variation of resistance for a $1 \%$ variation of the relative humidity. This sensitivity depends on the operating temperature of the sensor and on the relative humidity and is given by eq 3

$$
S=\left|\frac{\Delta R(\% \mathrm{RH}+10, T)-\Delta R(\% \mathrm{RH}, T)}{10}\right|
$$

The different values of sensitivity are listed in Table 1 . These sensitivity values are in the same range as other carbon-based materials, such as graphene, graphene oxide, etc., reported in the literature. $^{51}$

Calculation of Thermodynamics Constants: Langmuir's Model. To access the thermodynamic constants, we decided to model the kinetic transitory regime using Langmuir's model to describe the interaction between water molecules and the sensor surface. The hypotheses of this model are the following: a simple monolayer of adsorbed molecules without interaction between each other is present at the sensor surface in equilibrium with gaseous molecules. Although multilayer adsorption or island formation cannot be ruled out, we did not see any indications for processes besides single-layer adsorption, and the fitting based on Langmuir's model yielded high accuracies. The equilibrium constant of adsorption $K_{\mathrm{ads}}$ is defined as the ratio between the rate constants of adsorption $k_{\mathrm{A}}$ and desorption $k_{\mathrm{D}}$. At a given temperature, the fraction of occupied sites on the sensor surface is named $\theta$. The variation of $\theta$ is given by eq 4 , where $P_{\mathrm{H}_{2} \mathrm{O}}$ is the partial pressure of water in the gas phase

$$
\left(\frac{\mathrm{d} \theta}{\mathrm{d} t}\right)_{\mathrm{T}}=k_{\mathrm{A}} * P_{\mathrm{H}_{2} \mathrm{O}} *(1-\theta)-k_{\mathrm{D}} * \theta
$$

The expression of $\theta$ is given by solving the preceding equation, with $M$ as a constant

$$
\theta(t)=M^{*} \mathrm{e}^{-\left(k_{\mathrm{A}} * P_{\mathrm{H}_{2} \mathrm{O}}+k_{\mathrm{D}}\right) * t}+k_{\mathrm{A}} * P_{\mathrm{H}_{2} \mathrm{O}}
$$

Furthermore, we observe a change in resistivity when water is adsorbed on the surface of the sensor. The last hypothesis consists of the proportionality between the resistance $R(t)$, or 
A

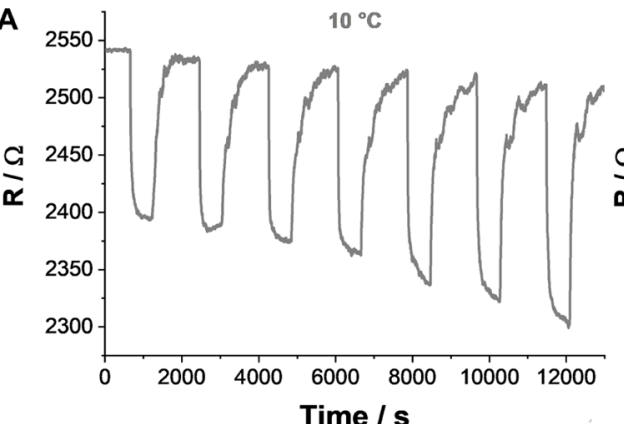

C

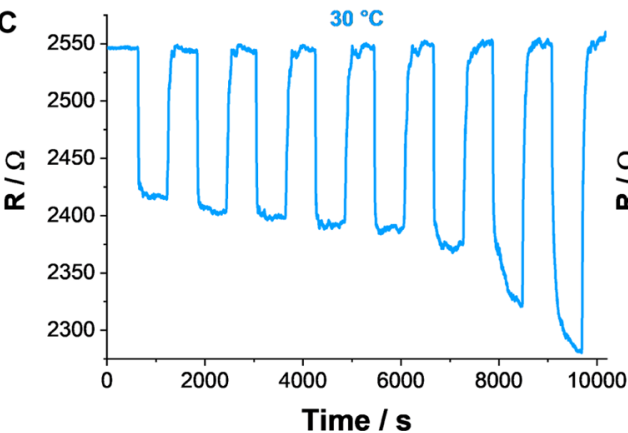

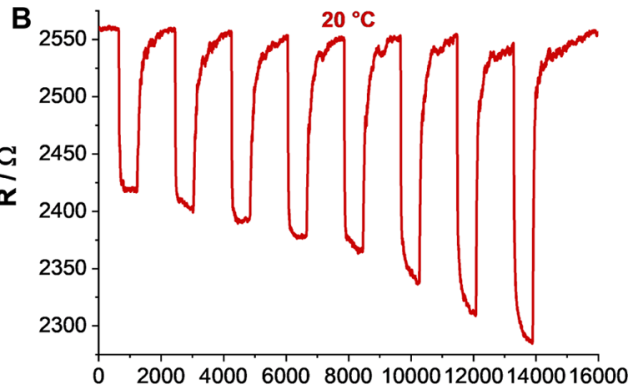

Time / s

$40^{\circ} \mathrm{C}$

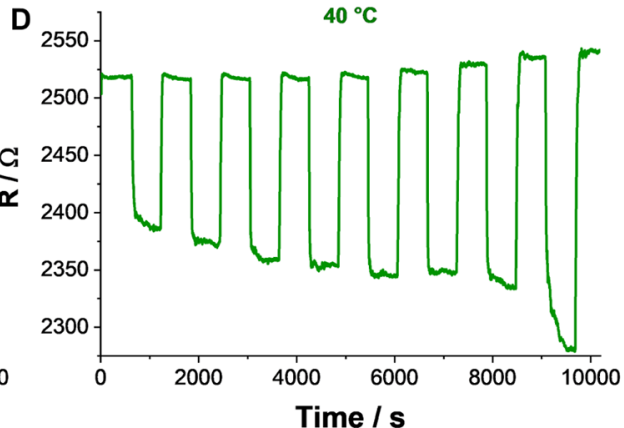

Figure 4. Variation of the sensor resistance during cycles of $10 \mathrm{~min}$ between dry air and different relative humidities at different temperatures. For each dataset, the relative humidity increases by $10 \%$ steps from 30 to $100 \%$ in steps of $10 \%$ (at $10{ }^{\circ} \mathrm{C}$ : from 40 to $100 \%$ ). At 10 and $20{ }^{\circ} \mathrm{C}$, the desorption cycles are $20 \mathrm{~min}$ long to completely reach the equilibrium.

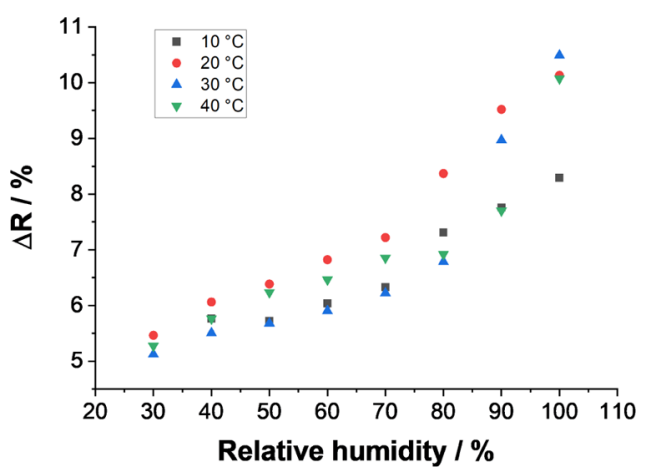

Figure 5. Relative variation of resistance $\Delta R$ with relative humidity at 10 (black squares), 20 (red circles), 30 (blue triangles), and $40{ }^{\circ} \mathrm{C}$ (green triangles).

Table 1. Sensitivity $S$ (in $\Omega \cdot \%$ ) of the Sensor at Different Temperatures for Different Intervals of Relative Humidity (\%RH)

\begin{tabular}{rrrrr} 
\%RH & $10{ }^{\circ} \mathrm{C}$ & $20{ }^{\circ} \mathrm{C}$ & $30{ }^{\circ} \mathrm{C}$ & $40{ }^{\circ} \mathrm{C}$ \\
$30-40$ & & 6.00 & 3.81 & 4.89 \\
$40-50$ & 0.39 & 3.23 & 1.75 & 4.72 \\
$50-60$ & 3.15 & 4.37 & 2.24 & 2.30 \\
$60-70$ & 2.91 & 3.98 & 3.18 & 3.90 \\
$70-80$ & 9.84 & 11.50 & 5.63 & 0.66 \\
$80-90$ & 4.51 & 11.51 & 21.86 & 7.81 \\
$90-100$ & 5.29 & 6.11 & 15.22 & 23.75 \\
\hline
\end{tabular}

more precisely, the corrected resistance $R^{\prime}(t)$ defined in eq 6, and the fraction of occupied sites by water on the surface. The proportionality coefficient between $R^{\prime}$ and $\theta$ is named $\alpha$ (eq 7).

$$
R^{\prime}(t)_{\% \mathrm{RH}, \mathrm{T}}=R(t)_{\mathrm{T}}-R(\% \mathrm{RH})_{\mathrm{T}}
$$

$$
\theta(t)=\alpha^{*} R^{\prime}(t)
$$

An example of the transitory regime of the corrected resistance $R^{\prime}$ during desorption is given in Figure 6 (black

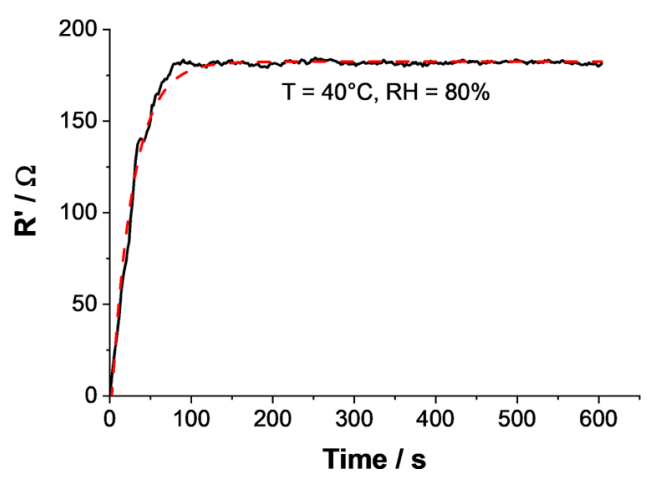

Figure 6. Kinetic transitory regime of the corrected resistance $R^{\prime}$ between two equilibrium states (black). At $t=0 \mathrm{~s}$, the sensor is in equilibrium in an atmosphere with $80 \%$ relative humidity and the chamber is flushed with dry air. The temperature is fixed at $40{ }^{\circ} \mathrm{C}$ during the entire experiment. The black curve was fitted based on the Langmuir model (red).

curve). At the beginning of the experiment, the sensor is in equilibrium at an atmosphere of $\mathrm{RH}=80 \%$ and $\mathrm{T}=40{ }^{\circ} \mathrm{C}$. At $t$ $=0 \mathrm{~s}$, the chamber is flushed with dry air, which causes the desorption of water molecules from the sensor surface. The resistance increases to reach a new equilibrium value in dry atmosphere. The corrected resistance $R^{\prime}$ is modeled (red curve) with an exponential law according to eq 5 . The rate constants $k_{\mathrm{A}}$ and $k_{\mathrm{D}}$ were determined from the model parameters to calculate the equilibrium constant $K_{\mathrm{ads}}$. All of the desorption curves at different relative humidities and different temperatures from Figure 4 were modeled by this approach. All values of fitted parameters and rate constants are 
available in Tables S1-S8. Notably, the response time of $\sim 100$ $\mathrm{s}$ is on the order of typical carbon-based materials but still rather slow compared to other materials, such as, e.g., Mxenes. $^{52-54}$

The evolution of the equilibrium constant $K_{\mathrm{ads}}$ for different relative humidities at $10,20,30$, and $40{ }^{\circ} \mathrm{C}$ is represented in Figure 7. For all of the tested atmospheric conditions, $K_{\mathrm{ads}}<1$,
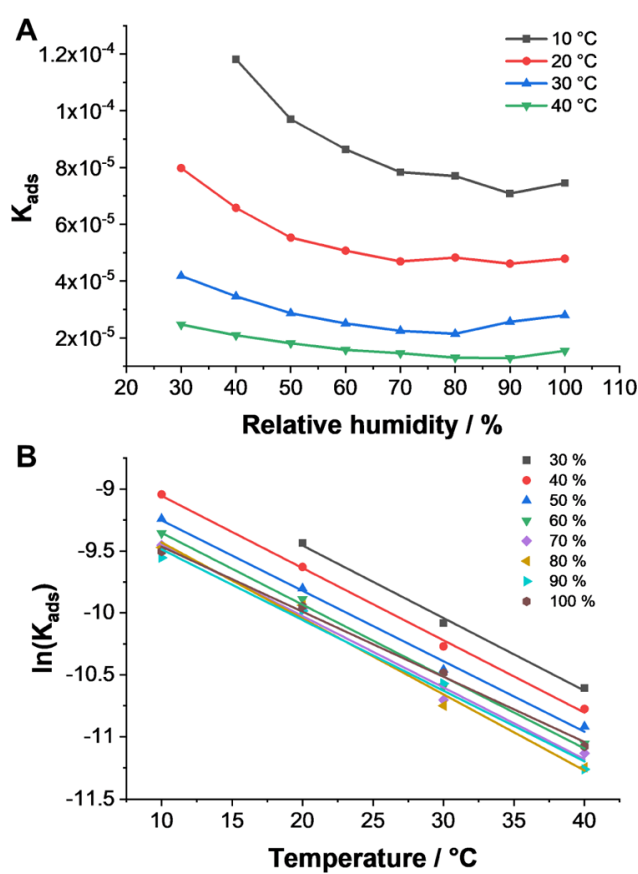

Figure 7. (A) Evolution of equilibrium constants $K_{\text {ads }}$ with relative humidity at different temperatures. (B) Plot of the logarithm of $K_{\mathrm{ads}}$ as a function of temperature at different relative humidities to determine the standard enthalpy of adsorption $\Delta_{\text {ads }} H$.

explaining why desorption is slower than adsorption (Figure 4, in particular, for 10 and $20{ }^{\circ} \mathrm{C}$ ). For atmospheres containing more than $70 \%$ of relative humidity, the equilibrium constant tends toward a constant value different for every temperature. This evolution suggests that the sensor surface begins to be saturated with $\mathrm{H}_{2} \mathrm{O}$ and the adsorption is slowing down. At a constant relative humidity, $K_{\text {ads }}$ decreases with temperature. This effect is well known and generally used to clean catalyst surfaces with high-temperature treatments.

Finally, it is possible to determine the standard enthalpy of water adsorption on the sensor surface according to the Van't Hoff equation (eq 8)

$$
\frac{\mathrm{d}\left(\ln K_{\mathrm{ads}}\right)}{\mathrm{d} T}=\frac{\Delta_{\mathrm{ads}} H^{\circ}}{\mathrm{RT}^{2}}
$$

Figure $7 \mathrm{~B}$ shows the variation of the logarithm of $K_{\mathrm{ads}}$ with temperature at different $\mathrm{RH}$ values. In this temperature range, straight lines are observed with the same slope (see Table S9 for details of linear regressions). An average value of the slope is used to determine the standard enthalpy of adsorption $\Delta_{\text {ads }} H$ using eq 8 . Thereby, $\Delta_{\text {ads }} H$ was estimated at $-42.6 \mathrm{~kJ} \cdot \mathrm{mol}^{-1}$. The sign of the standard enthalpy is negative because the water adsorption is favored on the sensor, explaining why $20 \mathrm{~min}$ of desorption is required at 10 and $20{ }^{\circ} \mathrm{C}$ compared to only 10 min of adsorption to reach the equilibrium. This value is in good agreement with those reported in the literature. ${ }^{55,56}$

\section{CONCLUSIONS}

A resistive humidity sensor has been fabricated using carbon laser patterning on a flexible substrate (PET) of our in-house developed carbon precursor ink. After laser irradiation, electronic microscopy (SEM and TEM), vertical scanning interferometry, Raman spectroscopy, and X-ray photoelectron spectroscopy show a porous foam-like morphology consisting of heteroatom-rich turbostratic graphitic carbon. This metalfree porous structure is conductive and shows semiconductorlike behavior with low positive Hall and Seebeck coefficients typically observed for carbon materials. All of these features entail temperature- and environment-dependent resistivity, making laser-patterned carbon suitable as a sensor platform.

A significant change in relative resistance of this small-scale sensor upon changing the relative humidity was measured with a high sensitivity between 1 and $10 \Omega \cdot \% \mathrm{RH}^{-1}$ depending on temperature and relative humidity. The sensor is usable under classical atmospheric conditions between 10 and $40{ }^{\circ} \mathrm{C}$ and in a broad range of relative humidity from 30 to $100 \%$. Finally, the kinetic transitory regime of water desorption has been studied at different temperatures and relative humidities. The equilibrium between gaseous and adsorbed water molecules is described with a simple Langmuir model, and equilibrium constants were calculated under different atmospheric conditions. The standard enthalpy of adsorption of water on the sensor carbonaceous surface is estimated at $42.6 \mathrm{~kJ} \mathrm{~mol}^{-1}$. Selective incorporation of Schottky junctions into the materials to improve the charge transfer kinetics and reduce the sensor dimensions is currently under investigation.

\section{EXPERIMENTAL SECTION}

Chemicals and Substrate. Citric acid (99.5\% for analysis) and urea (certified AR for analysis) were obtained from Fisher Scientific GmbH. Ethylene glycol ( $\geq 99.7 \%$, AnalaR Normapur) was obtained from VWR Chemicals. Polyvinylpyrrolidone (average mol. wt, $10000)$ was obtained from Sigma-Aldrich. Melinex sheets (PET substrates, $d=175 \mu \mathrm{m}$ ) were obtained from Plano $\mathrm{GmbH}$ and used as received.

Synthesis of the Carbon Network Forming Agent. Citric acid $(2.5 \mathrm{~g})$ and urea $(2.5 \mathrm{~g})$ were dissolved in $10 \mathrm{~mL}$ of distilled water in a crucible. The water was evaporated under vacuum to obtain a homogeneous mixture of solid citric acid and urea. The crucible was then heated to $300{ }^{\circ} \mathrm{C}$ under nitrogen for $2 \mathrm{~h}$ ( $1 \mathrm{~h} 30$ ramp time to reach $300{ }^{\circ} \mathrm{C}$ ) to obtain a black solid. This black solid was ground to a fine powder, dispersed in water, and stirred at $95{ }^{\circ} \mathrm{C}$ for $4 \mathrm{~h}$. The supernatant was removed by centrifugation. The washing process was repeated four times to fully remove free molecular species. The remaining black solid was dried under vacuum.

Ink and Film Preparation. A $200 \mathrm{~g} \cdot \mathrm{L}^{-1}$ solution of PVP (wt $10000)$ in ethylene glycol was prepared. This solution $(200 \mu \mathrm{L})$ and CAU300 (200 mg) were mixed in a $4 \mathrm{~mL}$ vial and stirred for at least 1 $\mathrm{h}$ to obtain a homogeneous black slurry. A homogeneous film of this ink was prepared by doctor blading on PET, and the ethylene glycol was evaporated at $80{ }^{\circ} \mathrm{C}$ on a hot plate.

Laser Patterning of the Sensor. Laser irradiation was realized with a Trotec Speedy 100 printer equipped with a $60 \mathrm{~W} \mathrm{CO}_{2}$ laser (10.6 $\mu \mathrm{m}$ wavelength). The laser was operated at $1 \mathrm{~W}$ and $17.6 \mathrm{~mm}$ $\mathrm{s}^{-1}$ in a pulsed mode at $1000 \mathrm{~Hz}$. These settings correspond to a fluence of $0.567 \mathrm{~J} \mathrm{~cm}^{-1}$. A typical resistive sensor platform was prepared as five $5 \mathrm{~mm}$ long lines separated by $0.1 \mathrm{~mm}$ and three concentric circles $(0.3,0.5$, and $0.7 \mathrm{~mm}$ diameter $)$ at both ends of the lines to ensure electric contact with silver ink (Figure 1A). Combustion analysis of the laser-patterned material was performed by removing the laser-patterned material from the substrate and subsequent washing in basic $\mathrm{H}_{2} \mathrm{O}$. 
Surface Area Measurement. The active surface area of the sensor was determined by the methylene blue adsorption method. $^{57-59}$ A laser-patterned carbon film of size $2 \times 1 \mathrm{~cm}^{2}$ was printed on PET sheets. The laser-patterned films were scratched off and collected, and their masses were determined with a microbalance $(\sim 0.3 \mathrm{mg})$. The powder was then dispersed in $9.5 \times 10^{-5} \mathrm{M}$ solutions of methylene blue in polypropylene vials and stirred for $24 \mathrm{~h}$. The solutions were centrifuged, and the amount of adsorbed MB was determined by measuring the absorbance of the supernatant with respect to a reference solution. An area of $1.35 \mathrm{~nm}^{2}$ per molecule MB is assumed. As a reference, the same mass of activated carbon was used $\left(1269 \mathrm{~m}^{2} \cdot \mathrm{g}^{-1}\right)$.

Humidity Sensing. The sensor was placed in a custom-built chamber with a gas flow inlet (Figure 1E). This chamber was thermalized in an ESPEC SH-641 environmental chamber from ATEC. The humidity flow was controlled by a humidity generator HUMIgen-04 from Dr. Wernecke GmbH. The gas flux was $80 \mathrm{~L} \cdot \mathrm{h}^{-1}$, and the relative humidity was controlled with a precision of $\pm 3 \%$.

Electrical characterizations were performed on a Solartron 1287 potentiostat in combination with an SI 1260 impedance unit. The frequency was fixed at $1000 \mathrm{~Hz}$, and the current intensity is $0.05 \mathrm{~mA}$ for all measurements.

Instrumental Section. Scanning electron microscopy was performed on a Zeiss LEO 1550-Gemini system (acceleration voltage: 3-10 kV). Transmission electron microscopy was performed using an EM 912 Omega from Zeiss operating at $120 \mathrm{kV}$. Raman spectra were obtained with a confocal Raman microscope (alpha300, WITec, Germany). The laser $(\lambda=532 \mathrm{~nm})$ was focused on the samples through a $20 \mathrm{x}$ objective. The laser power on the sample was set at 1.0 mW. X-ray diffraction was performed on a Bruker D8 Advance diffractometer in the Bragg-Brentano mode at the $\mathrm{Cu} \mathrm{K} \alpha$ wavelength. Vertical scanning interferometry was performed with a vertical scanning interferometer smartWLI compact (GBS mbH, Germany) with the software smartVIS3D 2.28 Tango and visualized with MountainsMap 8. Hall measurements were carried out at room temperature in an Accent HL5500PC using a magnetic strength of $0.3 \mathrm{~T}$. The samples were fabricated in a cloverleaf geometry, and the pins contacted the film via indium pads. After checking the ohmic response of the contacts, all of the measurements were done with the current source set for AC operation under vacuum. The entire Hall measurement was conducted five times, and the average value was sent to the result area. The whole procedure was repeated four times. The Seebeck coefficient was determined using a custom-built apparatus designed to implement the method of four coefficients. ${ }^{60}$ The Seebeck voltage is measured at four different temperature gradients, all near $298 \pm 3 \mathrm{~K}$. The thermopower is determined via linear fit on a plot of Seebeck voltage vs. temperature difference and is corrected for the built-in thermopower associated with the indium pads and copper blocks. The reported value is the average of four measurements. $X$-ray photoelectron spectra were recorded using a Kratos Axis Ultra DLD spectrometer equipped with a mono-spectra calibrated using carbon tape (Ted Pella) with a chromatic Al Ka X-ray source (hn 1/4 1486.6 $\mathrm{eV})$. The high-resolution $\mathrm{C}_{1 \mathrm{~s}}$ binding energy is $284.6 \mathrm{eV}$. Raw data were processed using CasaXPS software (version 2.3.23). C 1s spectra were fit using Gaussian-Lorentzian line shapes for all spectral components.

\section{ASSOCIATED CONTENT}

\section{SI Supporting Information}

The Supporting Information is available free of charge at https://pubs.acs.org/doi/10.1021/acsaelm.0c00942.

Materials characterization and data analysis (PDF)

\section{AUTHOR INFORMATION}

\section{Corresponding Author}

Volker Strauss - Department of Colloid Chemistry, Max-

Planck-Institute of Colloids and Interfaces, 14476 Potsdam,
Germany; ○ orcid.org/0000-0003-2619-6841;

Email: volker.strauss@mpikg.mpg.de

\section{Authors}

Simon Delacroix - Department of Colloid Chemistry, MaxPlanck-Institute of Colloids and Interfaces, 14476 Potsdam, Germany

Anna Zieleniewska - National Renewable Energy Laboratory,

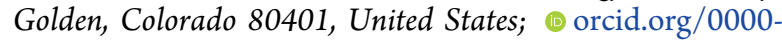
0001-9987-7520

Andrew J. Ferguson - National Renewable Energy Laboratory, Golden, Colorado 80401, United States

Jeffrey L. Blackburn - National Renewable Energy Laboratory, Golden, Colorado 80401, United States; (1) orcid.org/0000-0002-9237-5891

Sebastian Ronneberger - Department of Biomolecular Systems, Max-Planck-Institute of Colloids and Interfaces, 14476 Potsdam, Germany

Felix F. Loeffler - Department of Biomolecular Systems, MaxPlanck-Institute of Colloids and Interfaces, 14476 Potsdam, Germany; (ㅇ orcid.org/0000-0002-8227-2522

Complete contact information is available at:

https://pubs.acs.org/10.1021/acsaelm.0c00942

\section{Notes}

The authors declare no competing financial interest.

\section{ACKNOWLEDGMENTS}

The authors are grateful for financial support from the Max Planck Society, the Fonds der Chemischen Industrie, the German Federal Ministry of Education and Research (13XP5050A), and the Max-Planck-Fraunhofer cooperation (Glyco3Display). They thank Klaus Bienert for the help provided for the use of the humidity generator, the technicians of the Colloid Chemistry department for their assistance with materials characterizations, and Markus Antonietti for continuous educational support. This work was authored, in part, by the National Renewable Energy Laboratory, operated by Alliance for Sustainable Energy, LLC, for the U.S. Department of Energy (DOE) under Contract No. DEAC36-08GO28308. A.Z., A.J.F., and J.L.B. gratefully acknowledge funding provided by the Solar Photochemistry Program of the Chemical Sciences, Geosciences, \& Biosciences (CSGB) Division at the U.S. DOE Office of Science: Basic Energy Sciences. The views expressed in the article do not necessarily represent the views of the DOE or the U.S. Government.

\section{REFERENCES}

(1) Wongkaew, N.; Simsek, M.; Griesche, C.; Baeumner, A. J. Functional Nanomaterials and Nanostructures Enhancing Electrochemical Biosensors and Lab-on-a-Chip Performances: Recent Progress, Applications, and Future Perspective. Chem. Rev. 2019, 119, 120-194.

(2) Tripathi, K. M.; Kim, T. Y.; Losic, D.; Tung, T. T. Recent Advances in Engineered Graphene and Composites for Detection of Volatile Organic Compounds (VOCs) and Non-Invasive Diseases Diagnosis. Carbon 2016, 110, 97-129.

(3) Krishnan, S. K.; Singh, E.; Singh, P.; Meyyappan, M.; Nalwa, H. S. A Review on Graphene-Based Nanocomposites for Electrochemical and Fluorescent Biosensors. RSC Adv. 2019, 9, 8778-8781.

(4) Li, H.; Shi, W.; Song, J.; Jang, H. J.; Dailey, J.; Yu, J.; Katz, H. E. Chemical and Biomolecule Sensing with Organic Field-Effect Transistors. Chem. Rev. 2019, 119, 3-35. 
(5) Nathan, A.; Ahnood, A.; Cole, M. T.; Lee, S.; Suzuki, Y.; Hiralal, P.; Bonaccorso, F.; Hasan, T.; Garcia-Gancedo, L.; Dyadyusha, A.; et al. Flexible Electronics: The next Ubiquitous Platform. Proc. IEEE 2012, 100, 1486-1517.

(6) Rittersma, Z. M. Recent Achievements in Miniaturised Humidity Sensors-a Review of Transduction Techniques. Sens. Actuators, A 2002, 96, 196-210.

(7) Tulliani, J.-M.; Inserra, B.; Ziegler, D. Carbon-Based Materials for Humidity Sensing: A Short Review. Micromachines 2019, 10, 232.

(8) Lee, J.; Cho, D.; Jeong, Y. A Resistive-Type Sensor Based on Flexible Multi-Walled Carbon Nanotubes and Polyacrylic Acid Composite Films. Solid-State Electron. 2013, 87, 80-84.

(9) Yu, Y.; Zhang, Y.; Jin, L.; Chen, Z.; Li, Y.; Li, Q.; Cao, M.; Che, Y.; Yang, J.; Yao, J. A Fast Response-Recovery 3D Graphene Foam Humidity Sensor for User Interaction. Sensors 2018, 18, 4337.

(10) Schedin, F.; Geim, A. K.; Morozov, S. V.; Hill, E. W.; Blake, P.; Katsnelson, M. I.; Novoselov, K. S. Detection of Individual Gas Molecules Adsorbed on Graphene. Nat. Mater. 2007, 6, 652-655.

(11) Meng, Z.; Stolz, R. M.; Mendecki, L.; Mirica, K. A. ElectricallyTransduced Chemical Sensors Based on Two-Dimensional Nanomaterials. Chem. Rev. 2019, 119, 478-598.

(12) Ziegler, D.; Palmero, P.; Giorcelli, M.; Tagliaferro, A.; Tulliani, J.-M. Biochars as Innovative Humidity Sensing Materials. Chemosensors 2017, 5, 35 .

(13) Moon, H. G.; Jung, Y.; Shin, B.; Song, Y. G.; Kim, J. H.; Lee, T.; Lee, S.; Jun, S. C.; Kaner, R. B.; Kang, C.; et al. On-Chip Chemiresistive Sensor Array for On-Road NO x Monitoring with Quantification. Adv. Sci. 2020, 7, No. 2002014.

(14) Wang, F.; Swager, T. M. Diverse Chemiresistors Based upon Covalently Modified Multiwalled Carbon Nanotubes. J. Am. Chem. Soc. 2011, 133, 11181-11193.

(15) Ye, R.; James, D. K.; Tour, J. M. Laser-Induced Graphene: From Discovery to Translation. Adv. Mater. 2019, 31, No. 1803621.

(16) Han, T.; Nag, A.; Afsarimanesh, N.; Mukhopadhyay, S. C.; Kundu, S.; Xu, Y. Laser-Assisted Printed Flexible Sensors: A Review. Sensors 2019, 19, 1462.

(17) Inagaki, M.; Qiu, J.; Guo, Q. Carbon Foam: Preparation and Application. Carbon 2015, 87, 128-152.

(18) Strong, V.; Dubin, S.; El-Kady, M. F.; Lech, A.; Wang, Y.; Weiller, B. H.; Kaner, R. B. Patterning and Electronic Tuning of Laser Scribed Graphene for Flexible All-Carbon Devices. ACS Nano 2012, 6, 1395-1403.

(19) Lin, J.; Peng, Z.; Liu, Y.; Ruiz-Zepeda, F.; Ye, R.; Samuel, E. L. G.; Yacaman, M. J.; Yakobson, B. I.; Tour, J. M. Laser-Induced Porous Graphene Films from Commercial Polymers. Nat. Commun. 2014, 5, No. 5714.

(20) Ye, R.; James, D. K.; Tour, J. M. Laser-Induced Graphene. Acc. Chem. Res. 2018, 51, 1609-1620.

(21) Wang, Y.; Wang, Y.; Zhang, P.; Liu, F.; Luo, S. Laser-Induced Freestanding Graphene Papers: A New Route of Scalable Fabrication with Tunable Morphologies and Properties for Multifunctional Devices and Structures. Small 2018, 14, 1-9.

(22) Wang, F.; Wang, K.; Zheng, B.; Dong, X.; Mei, X.; Lv, J.; Duan, W.; Wang, W. Laser-Induced Graphene: Preparation, Functionalization and Applications. Mater. Technol. 2018, 33, 340-356.

(23) Sopronyi, M.; Sima, F.; Vaulot, C.; Delmotte, L.; Bahouka, A.; Ghimbeu, C. M. Direct Synthesis of Graphitic Mesoporous Carbon from Green Phenolic Resins Exposed to Subsequent UV and IR Laser Irradiations. Sci. Rep. 2016, 6, No. 39617.

(24) de Araujo, W. R.; Frasson, C. M. R.; Ameku, W. A.; Silva, J. R.; Angnes, L.; Paixão, T. R. L. C. Single-Step Reagentless Laser Scribing Fabrication of Electrochemical Paper-Based Analytical Devices. Angew. Chem., Int. Ed. 2017, 56, 15113-15117.

(25) Ye, R.; Chyan, Y.; Zhang, J.; Li, Y.; Han, X.; Kittrell, C.; Tour, J. M. Laser-Induced Graphene Formation on Wood. Adv. Mater. 2017, 29, No. 1702211.

(26) Chyan, Y.; Ye, R.; Li, Y.; Singh, S. P.; Arnusch, C. J.; Tour, J. M. Laser-Induced Graphene by Multiple Lasing: Toward Electronics on Cloth, Paper, and Food. ACS Nano 2018, 12, 2176-2183.
(27) Mahmood, F.; Zhang, C.; Xie, Y.; Stalla, D.; Lin, J.; Wan, C. Transforming Lignin into Porous Graphene via Direct Laser Writing for Solid-State Supercapacitors. RSC Adv. 2019, 9, 22713-22720.

(28) Strauss, V.; Anderson, M.; Turner, C. L.; Kaner, R. B. Fast Response Electrochemical Capacitor Electrodes Created by LaserReduction of Carbon Nanodots. Mater. Today Energy 2019, 11, 114119.

(29) Strauss, V.; Marsh, K.; Kowal, M. D.; El-Kady, M.; Kaner, R. B. A Simple Route to Porous Graphene from Carbon Nanodots for Supercapacitor Applications. Adv. Mater. 2018, 30, No. 1704449.

(30) Strauss, V.; Anderson, M.; Wang, C.; Borenstein, A.; Kaner, R. B. Carbon Nanodots as Feedstock for a Uniform Hematite-Graphene Nanocomposite. Small 2018, 14, No. 1803656.

(31) Strauss, V.; Wang, H.; Delacroix, S.; Ledendecker, M.; Wessig, P. Carbon Nanodots Revised: The Thermal Citric Acid/Urea Reaction. Chem. Sci. 2020, 11 (31), 8256-8266.

(32) Delacroix, S.; Wang, H.; Heil, T.; Strauss, V. Laser-Induced Carbonization of Natural Organic Precursors for Flexible Electronics. Adv. Electron. Mater. 2020, 6, No. 2000463.

(33) Malard, L. M.; Pimenta, M. A.; Dresselhaus, G.; Dresselhaus, M. S. Raman Spectroscopy in Graphene. Phys. Rep. 2009, 473, 51-87.

(34) Lazar, P.; Mach, R.; Otyepka, M. Spectroscopic Fingerprints of Graphitic, Pyrrolic, Pyridinic, and Chemisorbed Nitrogen in N-Doped Graphene. J. Phys. Chem. C 2019, 123, 10695-10702.

(35) Yamada, Y.; Kim, J.; Matsuo, S.; Sato, S. Nitrogen-Containing Graphene Analyzed by X-Ray Photoelectron Spectroscopy. Carbon 2014, 70, 59-74.

(36) Esquinazi, P.; Krüger, J.; Barzola-Quiquia, J.; Schönemann, R.; Herrmannsdörfer, T.; García, N. On the Low-Field Hall Coefficient of Graphite. AIP Adv. 2014, 4, No. 117121.

(37) Tu, N. D. K.; Lim, J. A.; Kim, H. A Mechanistic Study on the Carrier Properties of Nitrogen-Doped Graphene Derivatives Using Thermoelectric Effect. Carbon 2017, 117, 447-453.

(38) Jin, Z.; Yao, J.; Kittrell, C.; Tour, J. M. Large-Scale Growth and Characterizations of Nitrogen-Doped Monolayer Graphene Sheets. ACS Nano 2011, 5, 4112-4117.

(39) Ambrosi, A.; Chua, C. K.; Latiff, N. M.; Loo, A. H.; Wong, C. H. A.; Eng, A. Y. S.; Bonanni, A.; Pumera, M. Graphene and Its Electrochemistry - an Update. Chem. Soc. Rev. 2016, 45, 2458-2493.

(40) Itkis, M. E.; Pekker, A.; Tian, X.; Bekyarova, E.; Haddon, R. C. Networks of Semiconducting SWNTs: Contribution of Midgap Electronic States to the Electrical Transport. Acc. Chem. Res. 2015, 48, 2270-2279.

(41) Blackburn, J. L.; Kang, S. D.; Roos, M. J.; Norton-Baker, B.; Miller, E. M.; Ferguson, A. J. Intrinsic and Extrinsically Limited Thermoelectric Transport within Semiconducting Single-Walled Carbon Nanotube Networks. Adv. Electron. Mater. 2019, 5, No. 1800910

(42) Kaiser, A. B.; Skákalová, V. Electronic Conduction in Polymers, Carbon Nanotubes and Graphene. Chem. Soc. Rev. 2011, 40, 3786.

(43) Zahab, A.; Spina, L.; Poncharal, P.; Marlière, C. Water-Vapor Effect on the Electrical Conductivity of a Single-Walled Carbon Nanotube Mat. Phys. Rev. B 2000, 62, 10000-10003.

(44) Afify, A. S.; Ahmad, S.; Khushnood, R. A.; Jagdale, P.; Tulliani, J. M. Elaboration and Characterization of Novel Humidity Sensor Based on Micro-Carbonized Bamboo Particles. Sens. Actuators, B 2017, 239, 1251-1256.

(45) Zhang, X.; Ming, H.; Liu, R.; Han, X.; Kang, Z.; Liu, Y.; Zhang, Y. Highly Sensitive Humidity Sensing Properties of Carbon Quantum Dots Films. Mater. Res. Bull. 2013, 48, 790-794.

(46) Cunha, B. B.; Greenshields, M. W. C. C.; Mamo, M. A.; Coville, N. J.; Hümmelgen, I. A. A Surfactant Dispersed N-Doped Carbon Sphere-Poly(Vinyl Alcohol) Composite as Relative Humidity Sensor. J. Mater. Sci. Mater. Electron. 2015, 26, 4198-4201.

(47) Guo, L.; Jiang, H. B.; Shao, R. Q.; Zhang, Y. L.; Xie, S. Y.; Wang, J. N.; Li, X.; Bin; Jiang, F.; Chen, Q. D.; Zhang, T.; et al. TwoBeam-Laser Interference Mediated Reduction, Patterning and Nanostructuring of Graphene Oxide for the Production of a Flexible Humidity Sensing Device. Carbon 2012, 50, 1667-1673. 
(48) Yao, Y.; Chen, X.; Zhu, J.; Zeng, B.; Wu, Z.; Li, X. The Effect of Ambient Humidity on the Electrical Properties of Graphene Oxide Films. Nanoscale Res. Lett. 2012, 7, No. 363.

(49) Liu, L.; Tan, S. J.; Horikawa, T.; Do, D. D.; Nicholson, D.; Liu, J. Water Adsorption on Carbon - A Review. Adv. Colloid Interface Sci. 2017, 250, 64-78.

(50) At $10{ }^{\circ} \mathrm{C}, 30 \%$ of Relative Humidity Is Not Accessible with the Environmental Chamber.

(51) Lv, C.; Hu, C.; Luo, J.; Liu, S.; Qiao, Y.; Zhang, Z.; Song, J.; Shi, Y.; Cai, J.; Watanabe, A. Recent Advances in Graphene-Based Humidity Sensors. Nanomaterials 2019, 9, No. 422.

(52) An, H.; Habib, T.; Shah, S.; Gao, H.; Patel, A.; Echols, I.; Zhao, X.; Radovic, M.; Green, M. J.; Lutkenhaus, J. L. Water Sorption in MXene/Polyelectrolyte Multilayers for Ultrafast Humidity Sensing. ACS Appl. Nano Mater. 2019, 2, 948-955.

(53) Pang, Y.; Jian, J.; Tu, T.; Yang, Z.; Ling, J.; Li, Y.; Wang, X.; Qiao, Y.; Tian, H.; Yang, Y.; et al. Wearable Humidity Sensor Based on Porous Graphene Network for Respiration Monitoring. Biosens. Bioelectron. 2018, 116, 123-129.

(54) Zhou, G.; Byun, J.-H.; Oh, Y.; Jung, B.-M.; Cha, H.-J.; Seong, D.-G.; Um, M.-K.; Hyun, S.; Chou, T.-W. Highly Sensitive Wearable Textile-Based Humidity Sensor Made of High-Strength, Single-Walled Carbon Nanotube/Poly(Vinyl Alcohol) Filaments. ACS Appl. Mater. Interfaces 2017, 9, 4788-4797.

(55) Picaud, S.; Collignon, B.; Hoang, P. N. M.; Rayez, J. C. Adsorption of Water Molecules on Partially Oxidized Graphite Surfaces: A Molecular Dynamics Study of the Competition between $\mathrm{OH}$ and COOH Sites. Phys. Chem. Chem. Phys. 2008, 10, 6998-7009.

(56) Tarasevich, Y. I.; Aksenenko, E. V. Interaction of Water, Methanol and Benzene Molecules with Hydrophilic Centres at a Partially Oxidised Model Graphite Surface. Colloids Surf., A 2003, 215, 285-291.

(57) Naeem, S.; Baheti, V.; Wiener, J.; Marek, J. Removal of Methylene Blue from Aqueous Media Using Activated Carbon Web. J. Text. Inst. 2017, 108, 803-811.

(58) Till, P.; Brindley, G. W. Methylene Blue Absorption by Clay Minerals. In Determination of Surface Areas and Cation Exchange Capacities (Clay-Organic Studies XVIII); Pergamon Press: 1970; Vol. 18.

(59) Rafatullah, M.; Sulaiman, O.; Hashim, R.; Ahmad, A. Adsorption of Methylene Blue on Low-Cost Adsorbents: A Review. J. Hazard. Mater. 2010, 177, 70-80.

(60) Young, D. L.; Coutts, T. J.; Kaydanov, V. I.; Gilmore, A. S.; Mulligan, W. P. Direct Measurement of Density-of-States Effective Mass and Scattering Parameter in Transparent Conducting Oxides Using Second-Order Transport Phenomena. J. Vac. Sci. Technol., A 2000, 18, 2978-2985. 